\title{
Cardioprotective effect of vitamin $D$ and melatonin on doxorubicin-induced cardiotoxicity in rat model: an electrocardiographic, scintigraphic and biochemical study
}

\author{
Serdar Savaş Gül ${ }^{1} \odot$, Hatice Aygün² \\ ${ }^{1}$ Department of Nuclear Medicine, Gaziosmanpaşa University School of Medicine, Tokat, Turkey \\ ${ }^{2}$ Department of Physiology, Gaziosmanpaşa University School of Medicine, Tokat, Turkey
}

DOI: $10.18621 /$ eurj.410029

\begin{abstract}
Objectives: Doxorubicin (DOX) is an antineoplastic drug that is widely used in chemotherapy but its cardiotoxicity is the most important side effect that limits the clinical use of this drug. We investigated DOX treatment and the effects of vitamin D and melatonin on heart by electrocardiography, scintigraphic and biochemical methods.

Methods: In this study, forty-nine adult male Wistar albino rats $(220 \pm 15 \mathrm{~g})$ were randomly divided into seven groups ( $\mathrm{n}=7$ each), namely control $(\mathrm{CON}, \mathrm{n}=7$ ), doxorubicin (DOX, $\mathrm{n}=7$ ), melatonin (MEL, $\mathrm{n}=7$ ), vitamin $\mathrm{D}$ (Vit $\mathrm{D}, \mathrm{n}=7$ ), doxorubicin plus melatonin (DOX + MEL, $\mathrm{n}=7$ ), doxorubicin plus vitamin $\mathrm{D}$ ( DOX + Vit $\mathrm{D}$, $\mathrm{n}=7$ ), and doxorubicin plus melatonin and vitamin $\mathrm{D}(\mathrm{DOX}+\mathrm{MEL}+\mathrm{Vit} \mathrm{D}, \mathrm{n}=7$ ) groups. Cardiotoxicity was induced by intraperitoneal injection (i.p.) of DOX $\left(18 \mathrm{mg} / \mathrm{kg}\right.$, i.p.) on the $15^{\text {th }}, 16^{\text {th }}$ and $17^{\text {th }}$ days. Rats receiving vitamin D and melatonin treatment in the DOX-induced cardiotoxicity group received vitamin D $(60,000 \mathrm{IU} / \mathrm{kg}$, i.p.) were administered in a single dose and melatonin $(40 \mathrm{mg} / \mathrm{kg} / \mathrm{day}$, i.p.) for 17 days and were injected with (18 $\mathrm{mg} / \mathrm{kg}$, i.p.) on doxorubicin $15^{\text {th }}, 16^{\text {th }}$, and $17^{\text {th }}$ days. On the $18^{\text {th }}$ day electrocardiography (ECG), ${ }^{99 \mathrm{~m} T e c h n e t i u m ~ p y r o p h o s p h a t e ~ s c i n t i g r a p h y ~ a n d ~ b i o c h e m i c a l ~ p a r a m e t e r s ~ w e r e ~ a s s e s s e d . ~}$
\end{abstract}

Results: DOX caused changes in the ECG pattern, a significant decrease in heartbeat $(p<0.01)$, P wave $(p<$ $0.001)$ and QRS complex durations $(p<0.001)$, R wave amplitude $(p<0.001)$; elevation in ST-segment $(p<$ $0.001)$ and decrease in QT interval $(p<0,001)$, and R-R interval durations $(p<0.001)$; increase in the serum levels of cardiac injury markers (CK, BUN, cardiac troponin T), $(p<0.01)$, and increased ${ }^{99 \mathrm{~m} T e c h n e t i u m}$ pyrophosphate uptake $(p<0.001)$ as compared to the CON group. MEL, Vit D and MEL+Vit D administration showed a same protective effect against DOX-induced altered ECG pattern. Pre-treatment with MEL, Vit D and MEL+Vit D significantly protected the heart from the toxic effect of DOX, by decreasing the levels of of cardiac injury markers $(\mathrm{CK}, \mathrm{BUN}$, cardiac troponin T) $(p<0.001)$ and decreased the elevated level of ${ }^{99 m}$ Technetium pyrophosphate uptake $(p<0.001)$.

Conclusion: Vitamin D and melatonin treatment prevented all the parameters of DOX-induced cardiotoxicity in rats.

Keywords: doxorubicin-induced cardiotoxicty, vitamin D, melatonin, ${ }^{99 \mathrm{~m}}$ Technetium pyrophosphate

Received: March 27, 2018; Accepted: July 23, 2018; Published Online: September 12, 2018

Address for correspondence: Serdar Savaş Gül, MD., Gaziosmanpaşa University School of Medicine, Department of Nuclear Medicine, Tokat, Turkey E-mail: opnukleertip@gmail.com 
$\mathrm{D}$ oxorubicin (DOX) is an anthracycline derivative antibiotic commonly used for the treatment of leukemias, malignant lymphoma and various solid tumors. However, its clinical use is limited because of dose-dependent cardiotoxicity [1]. DOX causes various toxic effects, the most common of which is cardiotoxicity that can be divided into acute effects (electrocardiogram changes), late effect of DOX administration is cardiomyopathy and congestive heart failure [2].

The pathogenesis of DOX-induced cardiotoxicity is acted by different mechanisms; thought that oxidative stres induced cell injury, lipid peroxidation, mitochondrial damage, inflammation, and apoptosis play a role. However, the most popular hypothesis is that it should be noted that DOX increased free radical production and oxidative stress, play a major role in DOX-induced cardiotoxicity [3]. Oxidative stress is characterized as an imbalance between antioxidant defense systems and reactive oxygen species. DOX-induced oxidative stress has been shown to be inhibited by the administration of certain antioxidants to experimental animals $[2,4,5]$.

Melatonin as an important natural antioxidant, may reduce DOX-induced oxidative stress [6]. The protective effect of melatonin on cardiotoxicity induced by DOX has been demonstrated [2]. Ahmed et al. [7] demonstrated that treatment with melatonin could reduce DOX-induced cardiotoxicity by reduced oxidative stress and increased activity of the antioxidative enzymes.

Vitamin D (Vit D) plays regulatory activity in body function including the cardiovascular system. It is known that the pleiotropic effects of Vit D regulatory activation are responsible for the distribution of Vit D regulatory activity every where in the human body nervous system, intestine, kidney, bone, parathyroid gland, cardiovascular systemand myocardium [810]. A lot of study has shown that Vit D deficiency is significantly associated with cardiovascular disease $[11,12]$. Vit D deficiency leads to increase in serum parathyroid hormone (PTH) [13]. Increased PTH levels were reported to be associated with left ventricular hypertrophy and increased risk of heart failure [14]. In addition, treatment with Vit D decreased blood pressure in patients with hypertension and modified the heart failure, decrease in cardiovascular mortality [1517].
Although many previous studies have examined the role of Vit D in cardiovascular disease, accorfing to our knowledge none have studied with examined the effects of Vit D on DOX-induced cardiotoxicity. This study was conducted to investigate the potential protective effects of melatonin plus Vit $\mathrm{D}$ on DOX-induced cardiotoxicity manifested by changes in the ECG pattern, changes in biochemical parameters such as serum creatine kinase (CK), blood urea nitrogen (BUN), and cardiac troponin $\mathrm{T}(\mathrm{cTnT})$ and changes in ${ }^{99 \mathrm{~m}}$ Technetium pyrophosphate ${ }^{99 \mathrm{~m}} \mathrm{Tc}$ PYP) scintigraphy. ${ }^{99 \mathrm{~m} T c}$ PYP radiopharmaceuticals are involved in the necrotic tissue by binding to the calcium complex. It is routinely used in necrosis imaging in the nuclear medicine department. Additionally, our recent study has shown that combined melatonin plus Vit D treatment was superior to either one alone for protecting DOX-induced cardiotoxicity.

\section{METHODS}

\section{Study Design}

This is a randomized controlled experimental study.

\section{Animal Selection}

Forty-nine adult male Wistar albino rats $(220 \pm 15$ g) were kept seven per cage, under standardized conditions of equal periods of light and dark in a room with lighting control (12-hour light/dark cycle, $24 \pm$ $2^{\circ} \mathrm{C}$ ) and had free access to food and water. All the experimental procedures were approved by the Animal Ethics Committee of Gaziosmanpaşa University.

\section{Experimental Procedures}

The animals were randomly divided into 7 groups of 7 rats in each:

Group 1: Rats received standard laboratory diet and drinking water ad libitum, and received normal saline $5 \mathrm{ml} / \mathrm{kg}$ body weight intraperitoneal (i.p.) and served as normal control (CON) groups.

Group 2: Rats were injected with a DOX at a cumulative dose of $18 \mathrm{mg} / \mathrm{kg}$, i.p. at an interval of 24 $\mathrm{h}$ on the $15^{\text {th }}, 16^{\text {th }}$ and $17^{\text {th }}$ days and served as the doxorubicin (DOX) group.

Group 3: Rats were injected melatoninat a dose of 40 $\mathrm{mg} / \mathrm{kg} /$ day, i.p., (sigma, soluble in pure water) for 17 
days and served as the melatonin (MEL) group.

Group 4: The first day of the experiment, rats were administered in a single dose of vitamin D (60.000 IU $\mathrm{mg} / \mathrm{kg}$, i.p.) and served as the vitamin D (Vit D) group. Group 5: Rats received melatonin treatment (40 $\mathrm{mg} / \mathrm{kg} /$ day, i.p., sigma, soluble in pure water) for 17 days and were injected with doxorubicin (cumulative dose: $18 \mathrm{mg} / \mathrm{kg}$, i.p.) on the $15^{\text {th }}, 16^{\text {th }}$ and $17^{\text {th }}$ days and and served as the doxorubicin plus melatonin (DOX+MEL) group.

Group 6: The first day of the experiment, rats received a single dose of Vit D (60.000 IU mg/kg, i.p.) and were injected with doxorubicin (cumulative dose: $18 \mathrm{mg} / \mathrm{kg}$, i.p.) on the $15^{\text {th }}, 16^{\text {th }}$ and $17^{\text {th }}$ days and served as the doxorubicin plus vitamin D (DOX+Vit D) group.

Group 7: Rats received melatonin treatment (40 $\mathrm{mg} / \mathrm{kg} /$ day, i.p., sigma, soluble in pure water) for 17 days and the first day of the experiment, rats received a single dose of vitamin D (60.000 IU mg/kg/i.p) and were injected with DOX (cumulative dose: $18 \mathrm{mg} / \mathrm{kg}$, i.p.) on the $15^{\text {th }}, 16^{\text {th }}$ and $17^{\text {th }}$ days and served as the doxorubicin plus melatonin and vitamin D $(\mathrm{DOX}+\mathrm{MEL}+$ Vit D) group.

\section{Electrocardiography}

Anesthesia was assessed clinically by pedal reflex. Then, needle electrodes were inserted under the skin of the rats in lead II position. Electrocardiography (ECG) recordings were taken for 1 minutes using the acknowledge software (version 3.8) and the MP-150 multi-channel physiological analysis system (BioPac Systems Inc., USA). Changes in ECG pattern (amplitude of ST segment, R-amplitude, duration of P wave, QRS complex, QT interval, and R-R interval) were considered.

\section{Biochemical Assays}

At the end of the experimental period, all the rats were anesthetized under light anesthesia and blood was collected from the heart into Vacutainer serumseparated tubes. After $30 \mathrm{~min}$, the tubes were centrifuged at $1500 \times \mathrm{g}$ for $10 \mathrm{~min}$. Then, the clear serum was used for all following biochemical assays. CK, BUN, cTnT were estimated by kinetic determination using the commercial kits of Bechman by Bechman Coulter LX-2000 (Brea, CA, USA).

\section{Scintigraphic Images}

When experimental model $18^{\text {th }}$ days, $1 \mathrm{mCi}{ }^{99 m} \mathrm{Tc}$ PYP radiopharmaceutical was administered through i.p. in anesthetized rats. One hour after ${ }^{99 \mathrm{~m}} \mathrm{Tc}$ PYP radiopharmaceutical administration, static imaging with dual head gamma camera (E-CAM, Siemens, Germany) was performed in anterior and posterior positions with 2.55 zoom factor. The radionuclide uptake heart area was measured by drawing equal rectangular regions of interest (ROI) and ${ }^{99 \mathrm{~m} T c} \mathrm{PYP}$ uptake was calculated by semi-quantitative method to the all study groups.

\section{Statistical Analysis}

Statistical analyses on each parameter were performed using SPSS 20.0 program. Comparison of the groups was made by one-way analysis of variance (ANOVA), followed by the post hoc Tukey test. Pearson correlation analysis revealed a significant correlation between the ${ }^{99 \mathrm{~m}} \mathrm{Tc}$ PYP uptake ratio and serum levels of cardiac injury markers. The results are expressed as the means \pm standard error of mean (SEM). For all statistical tests, $p<0.05$ was considered statistically significant.

Table 1. Electrocardiographic parameters of the groups

\begin{tabular}{|c|c|c|c|c|c|c|c|}
\hline Groups & $\begin{array}{c}\text { Heart beat } \\
\text { (bpm) }\end{array}$ & $\begin{array}{c}\text { P wave } \\
\text { duration } \\
\text { (s) }\end{array}$ & $\begin{array}{c}\text { QRS } \\
\text { complex } \\
\text { duration } \\
\text { (s) }\end{array}$ & $\begin{array}{c}\text { QT interval } \\
\text { duration } \\
\text { (s) }\end{array}$ & $\begin{array}{c}R-R \text { interval } \\
\text { (s) }\end{array}$ & $\begin{array}{c}\text { R wave } \\
\text { amplitude } \\
(\mathrm{mV})\end{array}$ & $\begin{array}{l}\text { ST segment } \\
\text { amplitude } \\
(\mathrm{mV})\end{array}$ \\
\hline $\mathrm{CON}$ & $280 \pm 4$ & $0.034 \pm 0.01$ & $0.060 \pm 0.0$ & $0.064 \pm 0.01$ & $0.145 \pm 0.01$ & $0.91 \pm 0.2$ & $0.054 \pm 0.05$ \\
\hline DOX & $220 \pm 3^{c}$ & $0.022 \pm 0.02^{\mathrm{c}}$ & $0.035 \pm 0.0^{\mathrm{c}}$ & $0.086 \pm 0.02^{\mathrm{c}}$ & $0.242 \pm 0.01^{\mathrm{c}}$ & $0.52 \pm 0.2 \mathrm{c}$ & $0.174 \pm 0.06^{\mathrm{c}}$ \\
\hline MEL & $279 \pm 4$ & $0.033 \pm 0.02$ & $0.057 \pm 0.01$ & $0.065 \pm 0.02$ & $0.154 \pm 0.03$ & $0.93 \pm 0.2$ & $0.055 \pm 0.01$ \\
\hline ViTD & $274 \pm 2$ & $0.035 \pm 0.01$ & $0.058 \pm 0.01$ & $0.064 \pm 0.02$ & $0.140 \pm 0.01$ & $0.92 \pm 0.2$ & $0.050 \pm 0.02$ \\
\hline DOX+MEL & $251 \pm 4^{\mathrm{b}, \mathrm{e}}$ & $0.031 \pm 0.01^{\mathrm{f}}$ & $0.057 \pm 0.02^{\mathrm{f}}$ & $0.069 \pm 0.03^{\mathrm{f}}$ & $0.158 \pm 0.02^{\mathrm{f}}$ & $0.69 \pm 0.2^{\mathrm{c}, \mathrm{f}}$ & $0.059 \pm 0.01^{\mathrm{f}}$ \\
\hline DOX+ViTD & $253 \pm 5^{\mathrm{a}, \mathrm{f}}$ & $0.035 \pm 0.01^{\mathrm{f}}$ & $0.061 \pm 0.01^{\mathrm{t}}$ & $0.071 \pm 0.01^{\mathrm{e}}$ & $0.166 \pm 0.01^{\mathrm{f}}$ & $0.73 \pm 0.2^{\mathrm{c}, \mathrm{f}}$ & $0.063 \pm 0.01^{\mathrm{t}}$ \\
\hline DOX+VitD+MEL & $250 \pm 8^{\mathrm{b}, \mathrm{e}}$ & $0.033 \pm 0.0^{\mathrm{f}}$ & $0.060 \pm 0.01^{\mathrm{f}}$ & $0.068 \pm 0.01^{\mathrm{f}}$ & $0.142 \pm 0.01^{\mathrm{f}}$ & $0.70 \pm 0.2^{\mathrm{c}, \mathrm{f}}$ & $0.057 \pm 0.06^{\mathrm{f}}$ \\
\hline
\end{tabular}




\section{RESULTS}

\section{Electrocardiography}

The study show significant alteration of electrocardiographic patterns (duration of both $P$ wave and QRS complex, QT interval, R-R interval, and heart rate, amplitude of ST-segment, R-wave amplitude) in DOX-administered rats as compared to normal control rats. CON group showed a normal pattern on ECG, whereas the DOX-treated group showed an decreased heart beat $(p<0.01)$, P wave ( $p$ $<0.001)$ and QRS complex durations $(p<0.001)$, R wave amplitude $(p<0.001)$; elevation in ST-segment $(p<0.001)$ and decrease in QT interval $(p<0.001)$, and R-R interval durations ( $p<0.001)$ as compared to the CON group.
MEL pre-co-treatment in the DOX-treated group showed a decreased heart beat $(p<0.01)$ and $\mathrm{R}$ wave amplitude $(p<0.001)$, but did not show significant changes the other ECG parameters compared with the CON group. MEL pre-co-treatment in the DOXtreated group showed a icreased heart beat $(p<0.01)$, P wave $(p<0.001)$, QRS complex durations $(p<$ $0.001)$ and $\mathrm{R}$ wave amplitude $(p<0.001)$, decreased QT interval $(p<0.001)$, and R-R interval durations ( $\mathrm{p}$ $<0.001)$, ST-segment-amplitude $(p<0.001)$ was significant when compared to the DOX alone-treated group.

Vit D pre-co-treatment in the DOX-treated group showed a decreased heart beat $(p<0.05)$ and $\mathrm{R}$ wave amplitude $(p<0.001)$, but did not show significant changes the other ECG parameters compared with the

Table 2. The statistical analysis of the cardiac marker enzyme levels

\begin{tabular}{lccc}
\hline Groups & BUN & CK & cTnT \\
\hline CON & $17.11 \pm 0.59$ & $0.48 \pm 0.1$ & $3499 \pm 244$ \\
DOX & $183.77 \pm 2.99 \mathrm{c}$ & $3.87 \pm 0.13^{\mathrm{c}}$ & $10306 \pm 366 \mathrm{c}$ \\
MEL & $16.31 \pm 0.46$ & $0.41 \pm 0.01$ & $2175 \pm 34$ \\
ViTD & $22.5 \pm 0.98$ & $0.40 \pm 0.01$ & $2376 \pm 68$ \\
DOX+MEL & $84.75 \pm 14.41^{\mathrm{c}, \mathrm{f}}$ & $1.64 \pm 0.31^{\mathrm{c}, \mathrm{f}}$ & $5673 \pm 689^{\mathrm{c}, \mathrm{f}}$ \\
DOX+ViTD & $126.56 \pm 2.25^{\mathrm{c}, \mathrm{f}}$ & $2.18 \pm 0.41^{\mathrm{c}, \mathrm{f}}$ & $7336 \pm 641^{\mathrm{c}, \mathrm{f}}$ \\
DOX+VitD+MEL & $86.44 \pm 18.84^{\mathrm{c}, \mathrm{f}}$ & $2.11 \pm 0.45^{\mathrm{c}, \mathrm{f}}$ & $6089 \pm 681^{\mathrm{c}, \mathrm{f}}$ \\
\hline
\end{tabular}

$\mathrm{CON}=$ control, $\mathrm{DOX}=$ doxorubicin, $\mathrm{MEL}=$ melatonin, Vit $\mathrm{D}=$ vitamin $\mathrm{D}, \mathrm{DOX}+\mathrm{MEL}=$ doxorubicin plus melatonin, $\mathrm{DOX}+\mathrm{Vit} \mathrm{D}=$ doxorubicin plus vitamin $\mathrm{D}, \mathrm{DOX}+\mathrm{MEL}+\mathrm{Vit} \mathrm{D}=$ doxorubicin plus melatonin and vitamin $\mathrm{D}, \mathrm{BUN}=$ blood urea nitrogen, $\mathrm{CK}=$ creatine kinase, $\mathrm{cTnT}=$ cardiac troponin $\mathrm{T}$ ${ }^{\mathrm{a}} p<0.05,{ }^{\mathrm{b}} p<0.01,{ }^{\mathrm{c}} p<0.001$ all groups as compared to CON groups

${ }^{\mathrm{d}} p<0.05,{ }^{\mathrm{e}} p<0.01,{ }^{\mathrm{f}} p<0.001 \mathrm{DOX}+\mathrm{MEL}, \mathrm{DOX}+\mathrm{ViT} \mathrm{D}, \mathrm{DOX}+\mathrm{Vit} \mathrm{D}+\mathrm{MELgroups}$ as compared to DOX groups

Table 3. The statistical analysis of the scintigraphic data for the groups

\begin{tabular}{lc}
\hline Groups & Tc-99m PYP Uptake (cpm) \\
\hline CON & $52140 \pm 559$ \\
DOX & $279266 \pm 684^{\mathrm{c}}$ \\
MEL & $53946 \pm 987$ \\
ViTD & $54646 \pm 660$ \\
DOX+MEL & $185793 \pm 7073^{\mathrm{c}, \mathrm{f}}$ \\
DOX+ViTD & $212600 \pm 7550^{\mathrm{c}, \mathrm{f}}$ \\
DOX+VitD+MEL & $150431 \pm 13843^{\mathrm{c}, \mathrm{f}}$
\end{tabular}

$\mathrm{CON}=$ control, DOX $=$ doxorubicin, $\mathrm{MEL}=$ melatonin, Vit $\mathrm{D}=$ vitamin $\mathrm{D}, \mathrm{DOX}+\mathrm{MEL}=$ doxorubicin plus melatonin, $\mathrm{DOX}+\mathrm{V}$ it $\mathrm{D}=$ doxorubicin plus vitamin $\mathrm{D}, \mathrm{DOX}+\mathrm{MEL}+\mathrm{Vit} \mathrm{D}=$ doxorubicin plus melatonin and vitamin $\mathrm{D}$

${ }^{\mathrm{a}} p<0.05,{ }^{\mathrm{b}} p<0.01,{ }^{\mathrm{c}} p<0.001$ all groups as compared to CON groups

${ }^{\mathrm{d}} p<0.05,{ }^{\mathrm{e}} p<0.01,{ }^{\mathrm{f}} p<0.001 \mathrm{DOX}+\mathrm{MEL}, \mathrm{DOX}+\mathrm{ViT} \mathrm{D}, \mathrm{DOX}+\mathrm{Vit} \mathrm{D}+\mathrm{MEL}$ groups as compared to DOX groups 

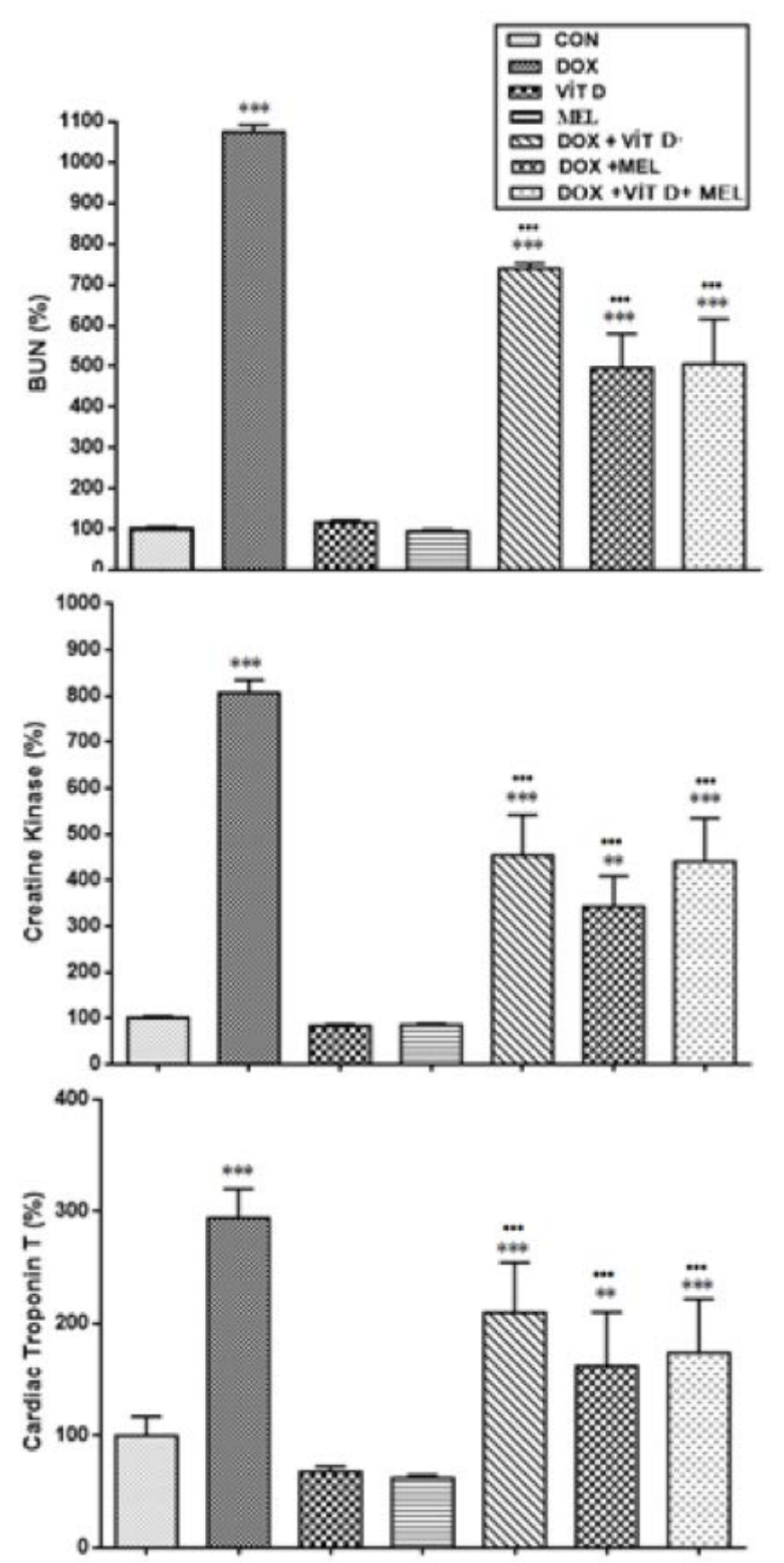

Figure 1. The effect of control (CON), doxorubicin (DOX), melatonin (MEL), vitamin D (Vit D), doxorubicin plus melatonin (DOX+MEL), doxorubicin plus vitamin D (DOX+Vit D), and doxorubicin plus melatonin and vitamin $\mathrm{D}(\mathrm{DOX}+\mathrm{MEL}+\mathrm{Vit} \mathrm{D})$ groups pre-treatment on the effect of cardiac marker enzymes, blood urea nitrogen (BUN), creatine kinase and cardiac troponin T. The DOX, DOX+MEL, DOX+ViT D and DOX+Vit D+MEL groups significantly increased the BUN, creatine kinase, cardiac troponin compared to control group $(* p<0.05, * * p<0.01$, *** $p$ $<0.001)$. Pre-treatment groups of DOX + MEL,DOX + ViT D and $\mathrm{DOX}+\mathrm{Vit} \mathrm{D}+\mathrm{MEL}$ significantly decreased the BUN, creatine kinase, cardiac troponin compared to DOX group $(p<0.05$, $(p<$ $0.01),(p<0.001)$. control group. Vit D pre-co-treatment in the DOXtreated group showed a icreased heart beat $(p<0.01)$, P wave $(p<0.001)$, QRS complex durations $(p<$ $0.001)$ and $\mathrm{R}$ wave amplitude $(p<0.001)$, decreased QT interval $(p<0.01)$, and R-R interval durations ( $p$ $<0.001)$, ST-segment-amplitude $(p<0.001)$ was significant when compared to the DOX alone-treated group.

$\mathrm{MEL}+$ Vit D+DOX pre-co-treatment in the DOXtreated group showed a decreased heart beat $(p<0.01)$ and $\mathrm{R}$ wave amplitude $(p<0.001)$, but did not show significant changes the other ECG parameters compared with the CON group. MEL+Vit D+DOX pre-co-treatment in the DOX-treated group showed an increased heart beat $(p<0.01)$, $\mathrm{P}$ wave $(p<0.001)$, QRS complex durations $(p<0.001)$ and $\mathrm{R}$ wave amplitude $(p<0.001)$, decreased QT interval $(p<$ $0.01)$ and $\mathrm{R}-\mathrm{R}$ interval durations $(p<0.001)$, STsegment-amplitude $(p<0.001)$ was significant when compared to the DOX alone-treated group (Table 1).

\section{Biochemical Assays}

DOX-treated group showed an increased BUN ( $p$ $<0.001)$, $\mathrm{CK}(p<0.001, \mathrm{cTnT}(p<0.001)$ as compared to the CON group. MEL, Vit D, MEL+Vit $\mathrm{D}+\mathrm{DOX}$ pre-co-treatment in the DOX-treated groups showed an increased BUN $(p<0.001), \mathrm{CK}(p<0.001$, cTnT $(p<0.001)$ as compared to the CON group. MEL, Vit D, MEL+Vit D+DOX pre-co-treatment in the DOX-treated groups showed an decreased BUN $(p<0.001)$, CK $(p<0.001$, cTnT $(p<0.001)$ were significant when compared to the DOX alone-treated group (Figure 1, Table 2).

\section{Scintigraphic Images}

${ }^{99 \mathrm{~m} T c}$ PYP scintigraphy images of the all study groups are shoen in Figure 2. DOX-treated group showed an increased ${ }^{99 \mathrm{~m}} \mathrm{Tc}$ PYP uptakeas compared to the CON group. MEL, Vit D, and DOX+MEL+Vit D pre-co-treatment in the DOX-treated groups showed an increased ${ }^{99 \mathrm{~m} T c}$ PYP radiopharmaceutical uptake $(p$ $<0.001)$ as compared to the CON group. MEL, Vit D, and DOX+MEL+Vit D pre-co-treatment in the DOXtreated groups showed an decreased ${ }^{99 \mathrm{~m}}$ Tc PYP uptake $(p<0.001)$ were significant when compared to the DOX alone-treated group (Figure 3, Table 3). 

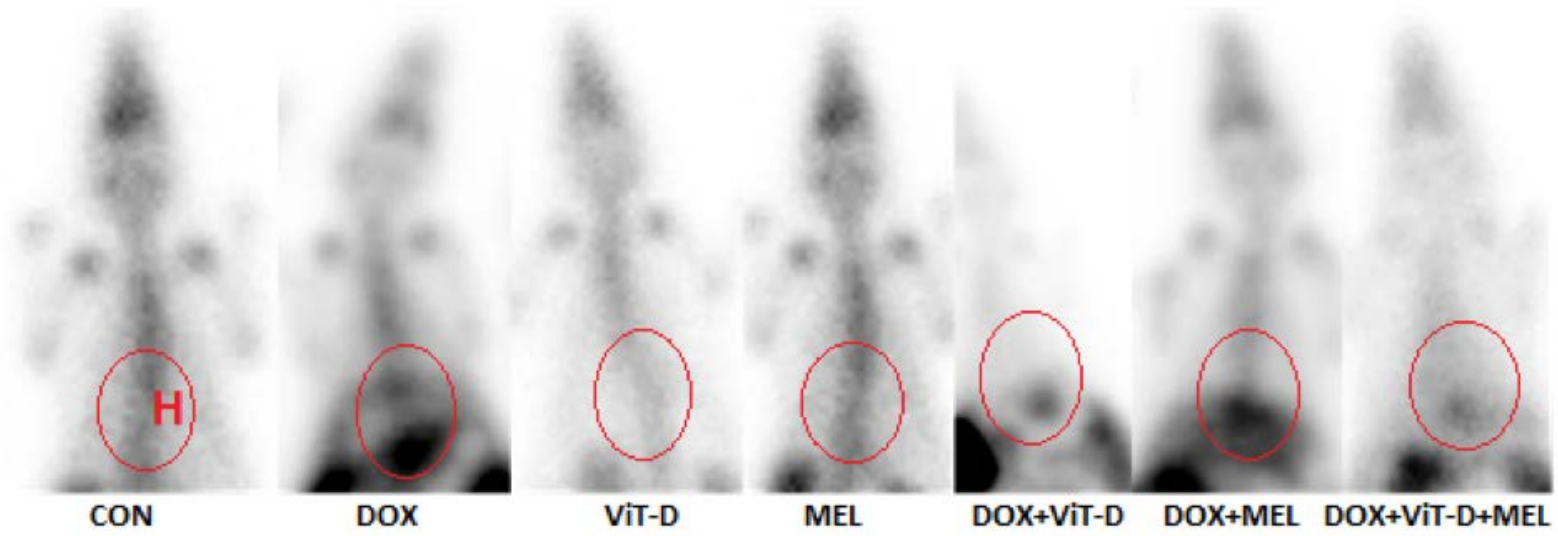

Figure 2. It is present that the ${ }^{99 \mathrm{~m}} \mathrm{Tc}$ PYP scintigraphic images of the all control (CON), doxorubicin (DOX), melatonin (MEL), vitamin D (Vit D), doxorubicin plus melatonin (DOX+MEL), doxorubicin plus vitamin D (DOX+Vit D), and doxorubicin plus melatonin and vitamin D (DOX+MEL+Vit D) groups. The radiopharmaceutical uptake was calculated for each rat by drawing equal rectangular ROI at heart area $(\mathrm{H})$.



Figure 3. The effect of control (CON), doxorubicin (DOX), melatonin (MEL), vitamin D (Vit D), doxorubicin plus melatonin $(\mathrm{DOX}+\mathrm{MEL})$, doxorubicin plus vitamin $\mathrm{D}(\mathrm{DOX}+\mathrm{Vit} \mathrm{D})$, and doxorubicin plus melatonin and vitamin $\mathrm{D}(\mathrm{DOX}+\mathrm{MEL}+\mathrm{Vit} \mathrm{D})$ groups on the effect of ${ }^{99 \mathrm{~m}} \mathrm{Tc}$ PYP radiopharmaceutical uptake. The DOX, DOX+MEL, DOX+ViT D andDOX+Vit D+MEL groups significantly increased the the ${ }^{99 \mathrm{~m} T c}$ PYP radiopharmaceutical uptake compared to control group $\left({ }^{*} p<0.05 * * p<0.01, * * * p<0.001\right)$. Pre-treatment groups of DOX+MEL,DOX+ViT D and DOX+Vit D+MEL significantly decreased the the ${ }^{99 \mathrm{~m}} \mathrm{Tc}$ PYP radiopharmaceutical uptake compared to DOX group $(p<0.05,(p<0.01),(p<0.001)$.

\section{DISCUSSION}

Previous studies demostrated that DOX treatmentinduced ECG abnormalities, which consist of non-specific $\mathrm{P}$ wave changes, sinus tachycardia, QRS complex, RR interval and $\mathrm{R}$ amplitude are frequently transient and cannot be said to be specific to anthracyclines. Prolonged QTc interval and elevation ST segment amplitude have been reported to be relatively characteristic ECG findings in receiving anthracyclines. The consecutive loss of cellular membrane damage due to oxidative stress might be 
characterized by ST elevation and prolonged QTc interval [18-21].

Our results confirmed that a cumulative dose of DOX $(18 \mathrm{mg} / \mathrm{kg})$ induces cardiotoxicity in rats as evidenced by ECG changes, ${ }^{99 \mathrm{~m} T c}$ PYP scintigraphic images and increased levels of cardiac biomarker enzymes. In the present study MEL and Vit D treated rats demonstrates several major findings regarding the effects of DOX-induced cardiotoxicity. First, we found that all of the ECG parameters changed significantly in the DOX-treated group as compared to the CON group, but MEL and Vit D restored the changes in ECG parameters in the DOX group to the pattern of the CON group. Second, cardiac marker enzymes (BUN, CK, cTnT) of the DOX-treated group increased significantly when compared with the CON group, whereas MEL and Vit D treatment could be attributed in part to the suppression of DOX-induced elevation in the levels of these cardiac marker enzymes. Third, ${ }^{99 \mathrm{~m}}$ Tc PYP scintigraphic images of the DOX-treated group was significantly changed as compared to the CON group, but MEL and Vit D treatment restored the changes in ${ }^{99 \mathrm{~m} T c}$ PYP scintigraphic images of the DOX group to the compared of the CON group.

In this study, ECG after DOX administration showed significant changes. DOX treatment induces a decrease $\mathrm{P}$ wave, QRS complex, in the amplitude of $\mathrm{R}$ wave, an increase in QT interval, $\mathrm{R}-\mathrm{R}$ interval and ST segment which may link to its degenerative effect on cell membrane $[22,23]$. Treatment with MEL, Vit $\mathrm{D}$ and combination MEL and Vit D groups caused increased in P-wave, QRS complex, $\mathrm{R}$ amplitude and decreased QT interval, R-R interval, ST segment was also near to normal. These changes in the electrocardiogram pattern induced by MEL and Vitamin D may be due to its membrane stabilizing action.

One of the major toxic effects of DOX is inducing lipid peroxidation. Its activation leads to acute membrane damage and releasing of cellular enzymes which decreased supply of oxygen to the myocardial cell leading to hypoxia [24-26]. The degree of DOXinduced cardiotoxicity can be estimated in serum and used as biomarkers to check the damage caused to the myocardium [27]. Several investigators reported that DOX causes elevation in the levels of CK-MB, BUN and cTnT serum biomarker activities after DOX administration $[28,29]$. In comparison to the CON group, the DOX-treated group showed significant elevation in the levels of cardiac marker enzymes in the serum. Treatment with MEL and Vit D caused a significant decrease in the levels of troponin T, BUN and CK-MB enzymes. This effect shows that MEL and Vit $\mathrm{D}$ can be prevent damage to the rat myocardium and stabilized the membrane.

As phosphorous compounds have high affinity for hydroxyapatite crystals, ${ }^{99 \mathrm{~m} T c}$ PYP scintigraphy has been widely used in the detection of bone tumors and metabolic bone diseases. ${ }^{99 \mathrm{~m} T c}$ PYP radiopharmaceutical accumulates predominantly as apatite-like crystals in mitochondria and cytoplasm of infarcted myocardial cells. Therefore, it has been considered beneficial in the diagnosis of acute phase myocardial infarction and quantification of the infarct size in the clinical setting [30-33]. Previous studies have also suggested that the degree of radiopharmaceutical accumulation such as ${ }^{99 \mathrm{~m} T c}$ labeled phosphonates, and ${ }^{99 \mathrm{~m}} \mathrm{Tc}$ PYP is a good index of the severity of tissue damage [34-38]. In the present study, we observed increased ${ }^{99 \mathrm{~m} T c}$ PYP radiopharmaceutical uptake on heart area in the DOXinduced cardiotoxicity rat model.

\section{Limitations}

This study was conducted to investigate the potential protective effects of melatonin plus Vit D on DOX-induced cardiotoxicity manifested by changes in the ECG pattern, changes in biochemical parameters and changes in ${ }^{99 \mathrm{~m} T c}$ PYP scintigraphy. Histopathological examination was not performed in this study.

\section{CONCLUSION}

The results of the present study revealed that subchronic and systemic administration of Vit D doses showed a considerable cardioprotective effect similar to MEL on DOX-induced cardiotoxicityin rat model. MEL is an antioxidant agent that prevents apoptosis in in experimental toxic animal models. Cardiotoxicity induced by DOX ECG findings are very variable and chemical biomarkers alone are not sufficient, therefore additional diagnostic methods are needed. As a result of the study, it was thought that MEL and Vit D administration may be effective in preventing DOX- 
induced cardiotoxicity and that ${ }^{99 \mathrm{~m}} \mathrm{Tc}$ PYP scintigraphy could be used in the follow-up of chemotherapy patients using DOX.

\section{Authorship declaration}

All authors listed meet the authorship criteria according to the latest guidelines of the International Committee of Medical Journal Editors, and all authors are in agreement with the manuscript.

\section{Conflict of interest}

The authors disclosed no conflict of interest during the preparation or publication of this manuscript.

\section{Financing}

The authors disclosed that they did not receive any grant during conduction or writing of this study.

\section{REFERENCES}

[1] Lefrak EA, Pitha J, Rosenheim S, Gottlieb JA. A clinicopathologic analysis of adriamycin cardiotoxicity. Cancer 1973;32:302-14.

[2] Bilginoğlu A, Aydın D, Ozsoy S, Aygün H. Protective effect of melatonin on adriamycin-induced cardiotoxicity in rats. Turk Kardiyol Dern Ars 2014;42:265-73.

[3] Swamy AH, Wangikar U, Koti BC, Thippeswamy AH, Ronad PM, Manjula DV. Cardioprotective effect of ascorbic acid on doxorubicin-induced myocardial toxicity in rats. Indian J Pharmacol 2011;43:507-11.

[4] Anjos Ferreira AL, Russell RM, Rocha N, Placido Ladeira MS, Favero Salvadori DM, Oliveira Nascimento MC, et al. Effect of lycopene on doxorubicin-induced cardiotoxicity: an echocardiographic, histological and morphometrical assessment. Basic Clin Pharmacol Toxicol 2007;101:16-24.

[5] Xin Y, Zhang S, Gu L, Liu S, Gao H, You Z, et al. Electrocardiographic and biochemical evidence for the cardioprotective effect of antioxidants in acute doxorubicininduced cardiotoxicity in the beagle dogs. Biol Pharm Bull 2011;34:1523-6.

[6] Othman AI, El-Missiry MA, Amer MA, Arafa M. Melatonin controls oxidative stress and modulates iron, ferritin, and transferrin levels in adriamycin treated rats. Life Sci 2008;83:563-8.

[7] Ahmed HH, Mannaa F, Elmegeed GA, Doss SH. Cardioprotective activity of melatonin and its novel synthesized derivates on doxorubicin-induced cardiotoxicity. Bioorg Med Chem 2005; 13:1847-57.

[8] Prufer K, Veenstra TD, Jirikowski GF, Kumar R. Distribution of 1,25-dihydroxyvitamin D3 receptor immunoreactivity in the rat brain and spinal cord. J Chem Immunol 1999;16:135-45.
[9] Langub MC, Herman JP, Malluche HH, Koszewski NJ. Evidence of functional vitamin D receptors in rat hippocampus. Neuroscience 2001;104:49-56.

[10] Walbert T, Jirikowski GF, Prufer K. Distribution of 1,25dihydroxyvitamin D3 receptor immunoreactivity in the limbic system. Horm Metab Res 2001;33:525-31.

[11] Kunadian V, Ford GA, Bawamia B, Qiu W, Manson JE. Vitamin D deficiency and coronary artery disease: a review of the evidence. Am Heart J 2014;167:283-91.

[12] Majumdar V, Prabhakar P, Kulkarni GB, Christopher R. Vitamin D status, hypertension and ischemic stroke: a clinical perspective. J Hum Hypertens 2015;29: 669-74.

[13] Holick MF, Binkley NC, Bischoff-Ferrari HA, Gordon CM, Hanley DA, Heaney RP, et al. Guidelines for preventing and treating vitamin D deficiency and insufficiency revisited. J Clin Endocrinol Metab 2012;97:1153-8.

[14] Bansal N, Zelnick L, Robinson-Cohen C, Hoofnagle AN, Ix JH, Lima JA, et al. Serum parathyroid hormone and 25hydroxyvitamin $\mathrm{D}$ concentrations and risk of incident heart failure: the Multi-Ethnic Study of Atherosclerosis. J Am Heart Assoc 2014;3:e01278.

[15] Park CW, Oh YS, Shin YS, Kim CM, Kim YS, Kim SY, et al. Intravenous calcitriol regresses myocardial hypertrophy in hemodialysis patients with secondary hyperparathyroidism. Am J Kidney Dis 1999;33:73-81.

[16] Shoji T, Shinohara K, Kimoto E, Emoto M, Tahara H, Koyama H, et al. Lower risk forcardiovascular mortality in oral 1-alpha-hydroxy vitamin D3 users in a haemodialysis population. Nephrol Dial Transplant 2004;19:179-84.

[17] Nemerovski CW, Dorsch MP, Simpson RU, Bone HG, Aaronson KD, Bleske BE. Vitamin D and cardiovascular disease. Pharmacotherapy 2009;29:691-708.

[18] Suzuki J, Yanagisawa A, Shigeyama T, Tsubota J, Yasumura T, Shimoyama K, et al. Early detection of anthracycline-induced cardiotoxicity by radionuclide angiocardiography. Angiology 1999;50:37-45.

[19] Kelishomi RB, Ejtemaeemehr S, Tavangar SM, Rahimian $\mathrm{R}$, Mobarakeh JI, Dehpour AR. Morphine is protective against doxorubicin-induced cardiotoxicity in rat. Toxicology 2008;243;96-104.

[20] Koti BC, Nagathan S, Vishwanathswamy A, Gadad PC, Thippeswamy A. Cardioprotective effect of Vedic Guard against doxorubicin-induced cardiotoxicity in rats: A biochemical, electrocardiographic, and histopathological study. Pharmacogn Mag 2013;9:176-81.

[21] Bhatt L, Joshi V. Mangifera indica L. leaf extract alleviates doxorubicin induced cardiac stress. J Intercult Ethnopharmacol 2017;6:284-9.

[22] Rossi F, Filippelli W, Russo S, Filippelli A, Berrino L. Cardiotoxicity of doxorubicin: Effects of drugs inhibiting the release of vasoactive substances. Pharmacol Toxicol 1994;75:99107.

[23] Holland RP, Brooks H. TQ-ST segment mapping: Critical review and analysis of current concepts. Am J Cardiol 1977;40:110-29.

[24] Villani F, Monti E, Piccinini F, Favalli L, Lanza E, Rozza 
Dionigi A, et al. Relationship between doxorubicin-induced ECG changes and myocardial alterations in rats. Tumori 1986;72:3239.

[25] Fujita T. Formation and removal of reactive oxygen species, lipid peroxides and free radicals, and their biological effects. Yakugaku Zasshi 2002;122:203-18.

[26] Zhang YW, Shi J, Li YJ, Wei L. Cardiomyocyte death in doxorubicin-induced cardiotoxicity. Arch Immunol Ther Exp (Warsz) 2009;57:435-45.

[27] Christenson ES, James T, Agrawal V, Park BH. Use of biomarkers for the assessment of chemotherapy-induced cardiac toxicity. Clin Biochem 2015;48:223-35.

[28] Ozdoğan K, Taşkın E, Dursun N. Protective effect of carnosine on adriamycin-induced oxidative heart damage in rats. Anadolu Kardiyol Derg 2011;11:3-10.

[29] Momin F, Shikalgar T, Naikwade N, Kalai B. Cardioprotective effect of methanolic extract of Ixora coccinea Linn. leaves on doxorubicin-induced cardiac toxicity in rats. Indian J Pharmacol 2012;44:178-83.

[30] Okuda K, Nohara R, Fujita M, Tamaki N, Konishi J, Sasayama S. Technetium-99m-pyrophosphate uptake as an indicator of myocardial injury without infarct. J Nucl Med 1994;35:1366-70.

[31] Okuda K, Nohara R, Ogino M, Tamaki N, Konishi J, Fujita $\mathrm{M}$, et al. Limitation of infarct size with preconditioning and calcium antagonist (diltiazem): difference in 99mTc-PYP uptake in the myocardium. Ann Nucl Med 1996;10:201-9.
[32] Kawano M, Taki J, Kinuya S, Higuchi T, Nakajima K, Miyazaki Y, et al. Improvement of 99mTc-pyrophosphate scintigraphy in detection of acute myocardial infarction: combined with 99mTc-tetrofosmin. Kaku Igaku 2001;38:707-13. [33] Mochizuki T, Murase K, Higashino H, Miyagawa M, Sugawara Y, Kikuchi T, et al. Ischemic "memory image" in acute myocardial infarction of 123I-BMIPP after reperfusion therapy: a comparison with 99mTc-pyrophosphate and 201Tl dual-isotope SPECT. Ann Nucl Med 2002;16:563-8.

[34] Affleck DG, Edelman L, Morris SE, Saffle JR. Assessment of tissue viability in complex extremity injuries: utility of the pyrophosphate nuclear scan. J Trauma 2001;50:263-9.

[35] Chang HR, Kao CH, Lian JD, Shu KH, Cheng CH, Wu MJ, et al. Evaluation of the severity of traumatic rhabdomyolysis using technetium-99m pyrophosphate scintigraphy. Am J Nephrol 2001;21:208-14.

[36] Matthews KL, Aarsvold JN, Mintzer RA, Chen CT, Lee RC. Tc-99m pyrophosphate imaging of poloxamer-treated electroporated skeletal muscle in an in vivo rat model. Burns 2006;32:755-64.

[37] Walker UA, Garve K, Brink I, Miehle N, Peter HH, Kelly T. 99 mTechnetium pyrophosphate scintigraphy in the detection of skeletal muscle disease. Clin Rheumatol 2007;26:1119-22.

[38] Çiftçi ÖD, Gül SS, Açıksarı K, Maman A, Çavuşoğlu T, Bademci R, et al. The diagnostic utility of scintigraphy in esophageal burn: a rat model. J Surg Res 2016;200:495-500. 